\title{
Vorwort Heft 3-2011
}

\author{
Hans-Christoph Grunau
}

Online publiziert: 4. August 2011

(C) Vieweg+Teubner und Deutsche Mathematiker-Vereinigung 2011

„Ricci-Fluss“ und „Bewertung von Finanzprodukten“ sind die gleichermaßen aktuellen wie unterschiedlichen Themenschwerpunkte des vorliegenden Heftes des Jahresberichts.

Gemäß einem klassischen Ergebnis von Heinz Hopf ist eine einfach zusammenhängende kompakte $n$-dimensionale Riemannsche Mannigfaltigkeit mit konstanter Schnittkrümmung 1 schon isometrisch zur Standardsphäre. Mit Blick auf dieses Ergebnis warf ebenfalls Heinz Hopf die Frage auf, ob solche Mannigfaltigkeiten mit Schnittkrümmungen im Intervall $(1-\varepsilon, 1]$ immerhin noch topologische Sphären seien. Weitergehend wurde diese Frage von Marcel Berger und Wilhelm Klingenberg 1960/61 positiv sogar für Schnittkrümmungen im Intervall $\left(\frac{1}{4}, 1\right]$ beantwortet; dieses Intervall ist auch optimal. Damit drängen sich aber sofort weitere Fragen auf, etwa ob ein analoges bzw. geeignet modifiziertes Resultat auch mit einer Diffeomorphieaussage, ohne die Voraussetzung einfachen Zusammenhangs oder bei nur punktweisen Schranken für das Verhältnis der Schnittkrümmungen zu beweisen sei. Simon Brendle diskutiert diesen Themenkomplex in seinem Übersichtsartikel „Der Sphärensatz in der Riemannschen Geometrie" und ordnet seine hier - zum Teil gemeinsam mit Richard Schoen - erzielten Ergebnisse in den Kontext des 1982 von Richard Hamilton eingeführten Ricci-Flusses ein. Simon Brendle widmet seinen Beitrag dem am 14.10.2010 verstorbenen Wilhelm Klingenberg.

Die Finanzkrisen der letzten Jahre haben deutlich vor Augen geführt, wie wichtig eine fundierte Bewertung und das Risikomanagement des Portfolios von Versicherungsgesellschaften für deren wirtschaftlichen Erfolg - oder oft sogar nur für deren wirtschaftliches Überleben - sind. Für eine fundierte mathematische Modellierung

H.-Ch. Grunau (凶)

Institut für Analysis und Numerik, Fakultät für Mathematik, Otto-von-Guericke-Universität, Postfach 4120, 39016 Magdeburg, Deutschland

e-mail: hans-christoph.grunau@ovgu.de 
und Theorie muss man finanzmathematische Konzepte in die klassische Versicherungsmathematik einführen. Dieses erläutern Thomas Knispel, Gerhard Stahl und Stefan Weber in ihrem Übersichtsartikel „From the equivalence principle to market consistent valuation“ und geben nach einer ausführlichen Erläuterung der Grundkonzepte und einer Einführung unter anderem in das Black-Scholes-Modell einen Überblick über moderne mathematische Instrumente zur Risikobewertung.

Die Buchbesprechungen diskutieren Neuerscheinungen zu Ruinwahrscheinlichkeiten sowie zu Gruppenwirkungen und Ergodentheorie. 